\title{
Optical Fiber Temperature Measurement for Process Industry
}

\author{
Ens, Wolfgang; Grieb, Herbert; Hilsendegen, Markus; Schorb, Herbert; von Dosky, Stefan \\ stefan.von_dosky@siemens.com \\ Siemens AG Karlsruhe, Germany
}

\begin{abstract}
:
The future of highly developed and cost-intensive facilities in the chemical industry is closely linked to innovative processes and plant technology. The requirements, in terms of reducing emissions and energy costs, mean that these "old" facilities must become specialized. Multiple educts to feed into a reactor, special temperature gradients and high pressures are often properties of new processes. Furthermore, little specific knowledge of plant operation is available because the development time from the laboratory to a completed production plant is short. In cases such as these, it makes sense to measure process temperature, for instance with a high spatial resolution. Temperature gradients and dangerous hot spots should be localized by using multipoint temperature sensors, [1]. Up until now, stainless steel tubes with integrated thermocouples or resistance thermometers are the devices of choice. Typically, a multipoint probe (lance) is a few meters long, about $6 \mathrm{~mm}$ in diameter and equipped with 8 thermocouples. To observe and control a larger reactor, a customer needs a lot of multipoints. The cabling is elaborate and lances such as these are subject to wear as a result of mechanical deformation, especially while they are being mounted. Furthermore, multipoint temperature measurement technology is not particularly versatile and cannot be employed for new applications.

This is the reason that an investigation was carried out to evaluate whether fiber optic measurement technologies have the required features and advantages to replace conventional multipoint thermometers. The properties of temperature value acquisition, such as resolution, precision, repeatability and long-term stability, must be comparable to multipoints. Further, fiber optic measurement technologies must be available at an attractive price. In any case, a far higher number of temperature sensors can be connected in series, while the lance volume is much lower, both suggesting that a fiber optic solution is attractive. Several existing measurement systems were analyzed and tested in the laboratory. The Fiber-Bragg-Grating (FBG) technology seems to be most mature for the applications being considered and industry.
\end{abstract}

The capabilities of alternative fiber optic measurement systems for different applications are first compared. For the preferred FBG technology, the properties of the established sensor and transmitter (interrogator) components are checked against classic temperature measurement techniques. Cross sensitivities to other physical phenomena [4], [5] are assessed, and finally a robust temperature measurement system for use in explosionprotected, high-pressure reactors is described. Furthermore, results of a long-term field test are presented.

Key words: Fiber-Bragg-Grating sensor, multipoint process temperature measurement, spatial distributed sensing 


\section{Selection of state-of-the-art fiber optic measurement systems}

For many years, fiber optic measurement systems have been used to detect certain physical effects along the fiber. Temperature, tension or the refractive index of a coupled medium (solid, liquid or gas) can be detected. In the basic setup, light from a source is coupled into the fiber and the reflected light is analyzed in terms of its frequency, amplitude or phase shifts. Specific physical effects lead to the following measurement signal changes.

- Fiber-Bragg-Grating (FBG): Interrogators typically consist of a polychromatic light source and a dispersive light sink (spectrometer). Each grating sensor is a short piece of fiber. Typically, at the position where the sensor is to be located on the fiber, the refractive index of the fiber core is segmented during production. A narrow band reflector, whose center frequency is shifted by the acting physical effect, is obtained. The signal amplitude can be used for grating diagnostics. The number of sensors per fiber depends mainly on the bandwidth of the interrogator, and is typically $<100 \mathrm{~nm}$. The fact that undesirable crosstalk can occur with close spectral gratings must also be taken into consideration.

- Optical Backscatter Reflectometry (OBR): This interrogation method combines FBG and OFDR. The amplitude of the backscattered light in an unmodified glass fiber control volume depends on small structural imperfections. Certain physical effects, e.g. the temperature, influence these imperfections. The local reflection amplitudes are detected, and compared to isothermal ones stored at a reference temperature. To improve the signal-to-noise ratio, conventional FBG structures can be inscribed (104 per fiber).

- Raman-based optical time domain reflectometry (OTDR): The sensor cable is a conventional glass fiber. The interrogator is equipped with a pulse laser source. The traveling time of the backscattered light allows for the localization of the measuring position and the Raman amplitude for the physical impact. The temperature can be calculated almost independent of e.g. mechanical stress, by evaluating both the Stokes and the anti-Stokes amplitudes.

- Raman-based optical frequency domain reflectometry (OFDR): The difference to the OTDR is that the localization is done in the frequency domain using an amplitude modulated laser source with sweepable modulation frequency. The frequency dependent backscattered Raman amplitudes are then converted back into the time domain for measuring point localization.

OBR is the most elaborate fiber interrogation technique for temperature measurement. Typical fiber lengths are $<500 \mathrm{~m}$. OTDR and OFDR are available both for the Raman and the Brillouin spectrum of the backscattered light. As the Brillouin technique comes with similar sensitivities for mechanical stress and temperature at the fiber segment, the Raman technique is mayor temperature sensitive. Each fiber segment length is $0.5 \ldots 2 \mathrm{~m}$ depending on the resolution of the interrogator and where the fiber segment is located (close to the interrogator or near the fiber end). Both interrogation techniques enable $>10000$ sensor segments per fiber, which can have an overall length of $50 \mathrm{~km}$. Typical applications are the mechanical and/or thermal observation of objects that extend over a wide area (pipelines, tunnels etc).

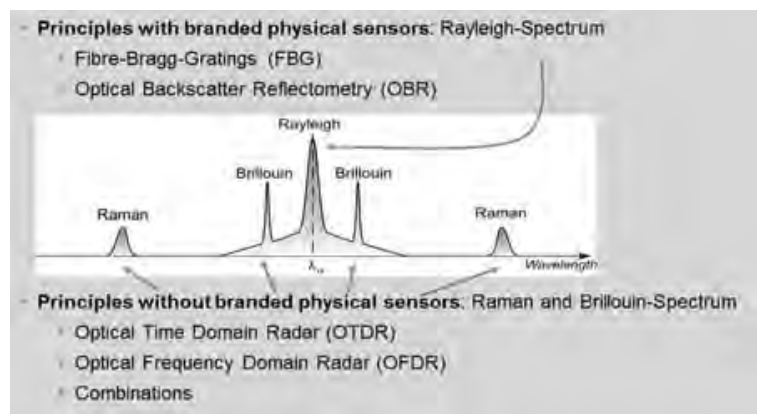

Fig. 1. Temperature measurement systems with sensors located along a glass fiber, spectral components of the backscattered light which are influenced by temperature

The most robust temperature measurement technique today with multiple glass fiber sensors is FBG. Technically, it has been known for decades and it enables the production of comparably mature interrogators based on standard telecommunication hardware in the $1550 \mathrm{~nm}$ range. Regarding the sensor, a rough differentiation can be made between manually produced standard FBGs and automatically produced draw tower grating FBGs (DTGs). With standard FBGs, the reflected light intensity is about 10 times higher. Like conventional glass fibers, DTGs must be equipped with a cladding that is attached to the coil while the fiber is being produced. Common claddings are based on polymers, which limit the upper temperature of DTG sensors to about $250^{\circ} \mathrm{C}$ in continuous operation. On the other hand, DTGs are comparably cheap as they can be automatically produced. 


\section{Configuration of the measurement system for the target industry}

The technical screening at the beginning of the project showed that with the appropriate technology, all the different systems can resolve the temperature to $<0.5 \mathrm{~K}$ in a range of approx. -270 to $600^{\circ} \mathrm{C}$. By employing FGBs in sapphire fibers, the upper temperature limit can be $>1000^{\circ} \mathrm{C}$. The properties of an FGB system perfectly match most of the requirements in the oil and gas and chemical industries. These requirements include a measuring range of -40 to $400^{\circ} \mathrm{C}$, a resolution of $0.1 \mathrm{~K}$, a sensor segment length $<100 \mathrm{~mm}$, no thermal crosstalk between sensor segments, length of the thermal profile to be measured is $<30 \mathrm{~m}$, accuracy $1^{\circ} \mathrm{C}$, optical power $<20 \mathrm{~mW}$ as a result of explosion protection stipulations, robustness and readout frequency, long-term stability and a price per sensor that is comparable with a standard single-point field device used in the process industry.

From a chemical industry point of view, there is no need for OTDR, OFDR or OBR systems. Furthermore, and considering the interrogator, it would be advantageous to develop a compact standard rail module that can be seamlessly integrated into an existing automation solution with a Profibus interface. As far as the sensor fiber is concerned, a measurement lance (fiber in a stainless steel capillary tube) complies with the requirements for most of the potential applications.

A $5 \mathrm{~m}$ lance with 25 DTGs was built to perform the first test. It was wound onto a $500 \mathrm{~mm}$ diameter reactor of a demonstration plant. The isothermal wavelengths of the sensors after installation were stored as reference values. Over a period of a few days, the reactor was filled, alternating with hot and cold water. After filling, the water was stirred and the temperature was measured at the same time using both the FBGs and a reference thermometer. The readout frequency was selected to be comparably low to prevent eventually existing mechanical crosstalk due to the water flow (swirls) around the lance. After a few days in operation and many temperature changes of the water, the variation in the readings from the 25 sensors (temperatures calculated from the FBG center wavelengths) was about $5 \mathrm{~K}$. The highest difference of one sensor to the reference RTD was 6K. These results were far from providing a practical benefit. With such an arrangement the fluid level could be measured, but the temperature could only be estimated.

One potential source for the drift was thought to be the mechanical interface involving the cladded fiber / inner surface of the capillary tube. This is the reason that alternative tubes with an inner diameter of $1 \mathrm{~mm}$ and $0.75 \mathrm{~mm}$ were tested. If small bending diameters (doglegs) are avoided, a fiber with an outer diameter of about 200 micron cannot be clamped macroscopically. The roughness of the inner capillary tube surface was tested to be $\mathrm{Rz}<20$ micron. Further, temperature tests were conducted with the lance mounted horizontally and vertically. In this case, the uncertainty of the measurement was lower, but irreversibility was still observed. Using an oil bath thermostat, the DTGs were tested at a maximum temperature of $150^{\circ} \mathrm{C}$.

The source of the irreversibility was estimated to be the compound fiber/cladding. The cladding polymer Ormocer [7] has a higher strain transfer coefficient to the steel tube than glass to a steel tube in the temperature range 60 to $85^{\circ} \mathrm{C}$ [6]. This results in a possible significant force transfer through contact with the combination Ormocer/steel, which is higher than for glass/steel. Furthermore, the thermal expansion coefficients of bare silica fiber (0.5E6 1/K) [8] and Ormocer (17E-6 1/K) are quite different. The expansion coefficient of the tested fibers remained unclear; however, it should differ from bare silica as a result of the dopants. Nevertheless, remaining differences to the cladding can generate mechanical stress inside the fiber itself. Because of the UV-based hardening process of the cladding after coating, a nonlinear contact with friction could be possible in individual segments of the lance. Summarizing, the detected hysteresis of the center wavelengths of DTGs in a tested temperature range up to $150^{\circ} \mathrm{C}$ is not acceptable for temperature sensors.

Parallel tests of an alternative lance with noncladded FBGs (3m long, 10 standard FBGs) did not manifest these negative effects. The drawback of this setup was considered to be the mechanical robustness of the lance. However, this assumption turned out not to be true. This is because over a test period of about two years at certain locations (installation and dismounting), no lance was damaged.

Two different approaches had been tested for the interrogators. One setup was based on a spectrometer light sink (where the light source is a super luminescence diode), and the second was based on a scanning laser light source (where the light sink is a photodiode). In addition to the readout frequency, which is of minor interest for temperature measurement, the technical parameters of both the devices were comparable. It was identified that the spectrometer-based interrogator has a warm up 
time of about three hours at room temperature until the measured center frequencies are constant. Until then, the values drifted about $15 \mathrm{pm}$, which is roughly $1.5 \mathrm{~K}$ with both a positive and a negative gradient. After this ramp up time no further frequency shift was identified over a period of a few days. The wavelength offset measured between two cold starts was up to $3 \mathrm{~K}$. Comparable magnitudes could be observed when changing the case temperature abruptly in varying directions (condensation was prevented). The reason for the drift was discussed; however, it seems to depend on the overall interrogator construction. On the contrary, the device with the laser source delivered stable center frequencies after about five minutes without significant offset in the same test scenarios. This is why it was the solution selected for further investigations.

Furthermore, a long-term stable wavelength/temperature reference for the interrogator was constructed. The module is equipped with some FBGs and a calibrated PT100 with a good thermal contact. The insulated module is mounted in the control cabinet close to the interrogator. Because the reference FBGs are not exposed to the process environment and only moderate temperature changes occur, a potential wavelength drift of the interrogator as well as diminishing light source amplitude (laser and/or photodiode) can be detected and automatically corrected. In addition, the polynomial approximation of the temperature has a better fit due to the very limited measurement range. The remaining uncertainty of the reference module (PT100 $30 \mathrm{mK}$ ) was not investigated but should be in the range of the resolution of the interrogator of $1 \mathrm{pm}$, which is approx. $0.1 \mathrm{~K}$.

\section{An excerpt of the measurements obtained}

A higher degree polynomial is used to obtain the temperature from the wavelength. Having a limited measurement range, a second degree polynomial is sufficient, [6]. If it is necessary to convert the wavelength in the temperature range -50 to $700^{\circ} \mathrm{C}$, which is the limit of the fiber lance, the approximation error is significant (refer to Fig. 2). This is the reason that a fourth degree polynomial was selected for the measurement system, which leads to minor systematical errors if the temperature is $>30^{\circ} \mathrm{C}$. The sampling points specified by the fiber supplier are statistically processed wavelength reference values.

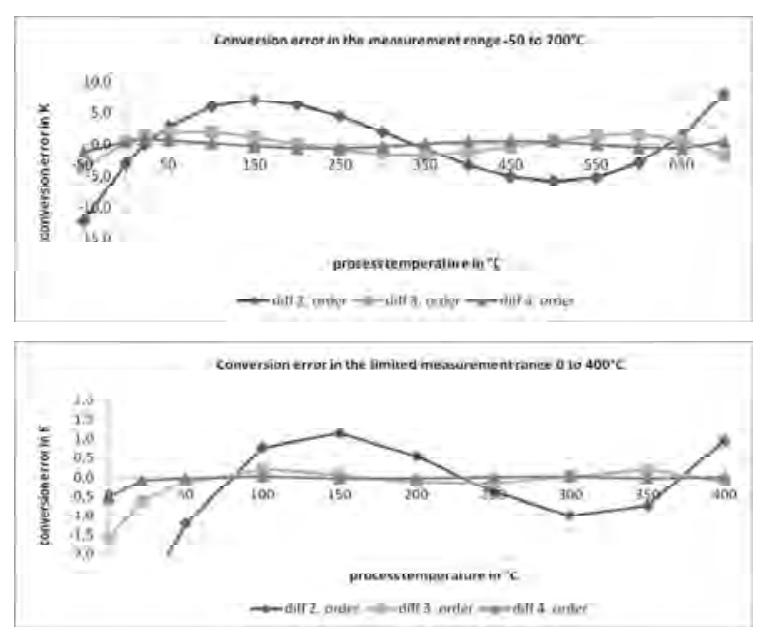

Fig. 2. Calculated systematical conversion error for the full and the limited measurement range

An excerpt of the measurement results of the system-swept laser interrogator/uncoated FBG lance are shown in Fig. 3.

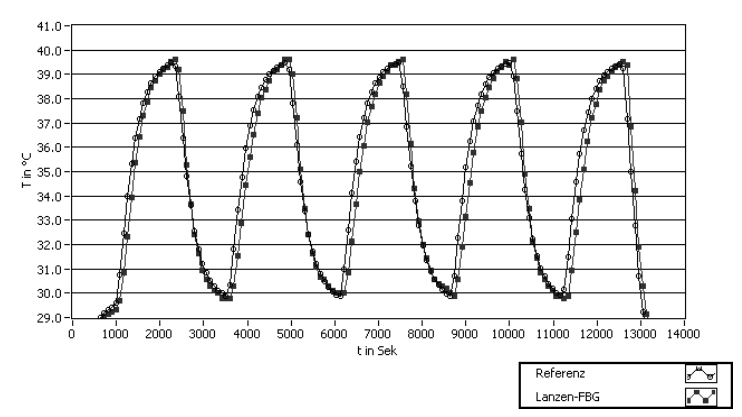

Fig. 3. Repeatability of one FBG of the temperature measurement system, reference PT100

Tests in a chemical production plant using a lance of $12.5 \mathrm{~m}$ and 22 FBGs over a period of several months at $260^{\circ} \mathrm{C}$ proved the robustness and the accuracy of the system. Furthermore, hot-spot tests were conducted with a heat gun on a vertically mounted lance. The heated segment of the capillary tube was subsequently cooled to the reference temperature using the oil thermostat. The measurement uncertainty of one FBG of the lance was determined to be +/$0.6 \mathrm{~K}$. The T90 time constant is $<4$ seconds.

Similar results were measured with the horizontally mounted lance. The lance was withdrawn and inserted repeatedly between the measurements to check if mechanical loads influence the offset of a measurement grating.

Summarizing, it can be stated that a zero point calibration adjusted in the factory is sufficient for using the measurement system at the customer plant. There is no need to calibrate the system on-site, if the customer has average requirements regarding measuring accuracy. 


\section{Literature}

[1] Siemens AG: Multipoint Thermometer

[2] IPHT Jena, www.ipht-jena.de

[3] Omnisens, www.omnisens.com

[4] Schlüter, V.G., Entwicklung eines experimentell gestützten Bewertungsverfahrens zur Optimierung und Charakterisierung der Dehnungsübertragung oberflächenapplizierter Faser-Bragg-Gitter-Sensoren, Dissertation TUBerlin, 2010

[5] www.ormocer.de

[6] Hoffmann, L. et. al., Applications of fibre optic temperature measurement 